\title{
Significance and characteristics of \\ Dexmedetomidine or Propofol-induced segmental electroencephalogram power spectra
}

Mao Zhou

department of anesthesiology,Sun Yat-Sen Memorial Hospital, Sun Yat-Sen University

Jing Wen

department of anesthesiology, Sun Yat-Sen Memorial Hospital, Sun Yat-Sen Univerisity.

Jun Peng

department of anesthesiology, Sun Yat-sen Memorial Hospital, Sun Yat-sen University.

Zhiyi Zhi ( $\nabla$ zz3c@virginia.edu )

department of anesthesology, University of Virginia

Shuling Peng ( $\nabla$ pslmzk@aliyun.com )

Sun Yat-Sen Memorial Hospital, Sun Yat-sen University Department of anesthesiology

Research article

Keywords:

Posted Date: March 28th, 2020

DOl: https://doi.org/10.21203/rs.3.rs-18956/v1

License: (9) This work is licensed under a Creative Commons Attribution 4.0 International License.

Read Full License 


\section{Abstract}

Background: Although the electroencephalogram patterns induced by dexmedetomidine and propofol are relatively similar, these drugs may have different molecular targets and involvement of distinct neural circuit dynamics in their effects. Our aim was to identify special neurophysiology signatures induced by these drugs.

Methods: Forty seven patients receiving combined spinal epidural anesthesia (CSEA®were randomly divided into two groups: Dexmedetomidine group ( $D$ group, $n=24$ ) and Propofol group (P group, $n=23$ ). Dexmedetomidine at $1 \mu \mathrm{g} / \mathrm{kg}$ was administered intravenously in $10 \mathrm{~min}$, then adjusted to a rate of 0.5 $\mu \mathrm{g} / \mathrm{kg} / \mathrm{h}$ and withheld if Ramsey sedation scale (RSS) reached 4 - 5 . Propofol was given via a computercontrolled infusion to target a concentration of $0.5 \mu \mathrm{g} / \mathrm{mL}$ at the effect site and then to gradually increase the concentration $0.5 \mu \mathrm{g} / \mathrm{mL}$ every $5 \mathrm{~min}$ until satisfactory sedation was achieved. The vital signs, segmental Narcotrend EEG power spectra and arterial blood analysis results were recorded before sedation, and 0, 5, 10 and 15 min after drug administration ended.

Results: After satisfactory sedation was achieved (RSS 4 - 5), propofol sedation was characterized with alpha wave, and dexmedetomidine sedation was characterized with theta wave.; After drug administration ended, relative power in alpha and beta waves of $\mathrm{P}$ group was higher than $\mathrm{D}$ group $\mathbb{D}]$ 0.05); However, when patients were croused or patted on their shoulders, relative power in alpha, beta and theta was increased $₫ \nabla \otimes 0.05$ ) and relative power in delta wave was decreased $\otimes P \otimes 0.05 \bigotimes$ in $D$ group; The percentages of alpha and theta power were decreased gradually at drug withdrawal, $5 \mathrm{~min}, 10 \mathrm{~min}, 15 \mathrm{~min}$ after drug administration ended; Narcotrend data have a good correlation with propofol sedation depth compared with the traditional sedation score.

Conclusion: Distinct EEG patterns are induced by propofol and dexmedetomidine. Since patients sedated with dexmedetomidine may be easier to be waked up than those with propofol, this phenomenon may be associated with the lower alpha power percentage, a more easily blocked alpha wave and a higher excitability of delta wave under dexmedetomidine sedation.

\section{Key Points}

Question: Are there any special neurophysiological signatures induced by these drugs that can be observed from unprocessed 1-channel EEG from the front of the head that can be widely used in clinical setting?

Findings: Patients sedated with dexmedetomidine may be easier to be waked up than those with propofol, this phenomenon may be associated with the lower alpha power percentage, a more easily blocked alpha wave and a higher excitability of delta wave under dexmedetomidine sedation.

Meaning: Distinct EEG patterns are induced by propofol and dexmedetomidine, Narcotrend data have a good correlation with propofol sedation depth compared with the traditional sedation score. 


\section{Introduction}

Electroencephalogram (EEG) has been widely used for nearly 20 years in anesthesia practice. Indices derived from EEG are the most widespread approach to monitor the depth of anesthesia ${ }^{1}$. In general, these indices computed from regression methods link the EEG features to the clinical states of the brain in sedation and general anesthesia. According to the scaled values from 0 to 100 , the indices indicate arousal levels from awake to different depth of anesthesia ${ }^{2}$. In addition, EEG has been marked for helping decrease unintended intraoperative awareness. However, its usage as an indispensable anesthesia monitoring technique has been questioned. There are three main issues, first, the use of electroencephalogram-produced indices to guide anesthetic use does not eradicate awareness under general anesthesia. Second, EEG indices do not reflect directly the neural electrical activities of how a special anesthetic exert its effects in the brain. Finally, general anesthetics or sedative agents may have different molecular mechanisms for their actions and may induce different behavioral properties that are likely accomplished by distinct neural circuit dynamics ${ }^{3-5}$. These complexities cannot be accurately reflected by a simple number between 0 and 100 as currently used.

Relatively similar EEG patterns can be seen between the $y$-Aminobutyric acid receptor-specific agonist propofol and the a2-adrenoceptor agonist dexmedetomidine. However, their molecular targets in neural circuits and behavioral characteristics are very different ${ }^{6}$. Studies of the 64-channel electroencephalogram changes caused by dexmedetomidine and propofol in healthy volunteers have shown that dexmedetomidine, at clinical dose, gave rise to a state similar to non-rapid eye movement sleep (NREM), in which volunteers are easily arousable and minimal in respiratory depression, while slowdelta and alpha oscillations are the markers of propofol-induced unconsciousness at high doses 7,8 . These differences beg the questions: 1) Are there any special neurophysiological signatures induced by these drugs that can be observed from unprocessed 1-channel EEG from the front of the head that can be widely used in clinical setting? 2) If special markers can be detected in the EEG, can these signatures induced by these drugs explain the observed differences in clinical response and behavior? 3) Would these drug-induced specific signatures allow anesthesiologists to make more detailed and accurate assessments of anesthesia depth than those based on EEG indices?

To explore these questions, we measured and compared the unprocessed EEG induced by dexmedetomidine and propofol using 1-channel (channel-1) Narcotrend in patients who received elective lower abdominal surgery or lower extremity surgery under epidural-spinal anesthesia plus sedation.

\section{Methods}

Study Design and Subjects

This study was approved by the Human Research Committee at the Sun Yat-Sen Memorial Hospital

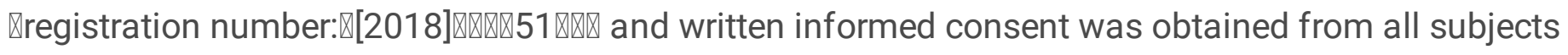
participating in the trial. The trial was registered prior to patient enrollment at clinicaltrials.gov 
(registration number: ChiCTR1900021562, Principal investigator: Shuling Peng, MaoZhou, Date of registration: 2019.05).

\section{Patient selection and data collection}

Forty-seven patients aged $18-40$ years who were going to receive elective lower abdominal surgery or lower extremity surgeries under combined spinal-epidural anesthesia $₫ C S E A \rrbracket$ were enrolled in the study. All subjects had a detailed preoperative evaluation. Patients who met all the inclusion criteria (American Society of Anesthesiologists (ASA) Grade I and II status, 18 to 40 years of age and gave informed written consent) were enrolled in the study. Exclusion criteria included obesity with a body mass index $\mathbb{\nabla} 30$ $\mathrm{kg} / \mathrm{m} 2$, pregnancy and drug or alcohol abuse. Subjects were not on any chronic medications including anticonvulsants, antidepressants or other psychoactive medications. Subjects were randomly divided into two groups: the dexmedetomidine group ( $D$ group, $n=24)$ and the propofol group ( $P$ group, $n=23)$. The study was approved by the Human Research Committee at the Sun Yat-Sen Memorial Hospital (Guangzhou, China). All subjects provided written informed consent. The study was conducted according to good clinical practice guidelines and was registered in Chinese Clinical Trial Registry (ChiCTR) (www.chictr.org.cn, registration number: ChiCTR1900021562).

\section{Combined spinal-epidural anesthesia and mode of administration}

All pre-anesthetic evaluations of patients were performed by an anesthesiologist one day before the surgery. In the operating theater, a peripheral intravenous access was secured using an $18 \mathrm{G}$ cannula and ASA standard monitors were attached. Blood pressure (BP), electrocardiograph (ECG), pulse rate, and $\mathrm{S}_{\mathrm{P}} \mathrm{O}_{2}$ were recorded. As a standard premedication, $10 \mathrm{mg}$ metoclopramide was given intravenously. All patients were preloaded with Ringer lactate solution $20 \mathrm{ml} / \mathrm{kg}$ before the block. Intravenous fluids were given intraoperatively based on body weight and requirements for surgery loss.

Patients were placed in a sitting position. An epidural catheter of 18-gauge was placed in the epidural space at L2-L3 interspace under aseptic precaution and with midline approach. A test dose of $3 \mathrm{ml}$ of $0.5 \%$ lidocaine with adrenaline 1:200,000 was administered to exclude intrathecal or intravascular placement of the catheter.

Using a 24-26-gauge Quincke spinal needle, subarachnoid block was performed at L3-L4 interspace and $3 \mathrm{ml}$ of $0.5 \%$ isobaric levobupivacaine was administered for both groups. Intraoperative block characteristics, such as time taken for motor block, time taken to reach T10 dermatome, and maximum level of block, were recorded.

Electroencephalogram preprocessing and epoch selection

After the combined spinal-epidural anesthesia (CSEA) was established, dexmedetomidine was administered with a $1 \mu \mathrm{g} / \mathrm{kg}$ loading bolus over $10 \mathrm{~min}$, followed by a $0.5 \mu \mathrm{g} / \mathrm{kg} / \mathrm{h}$ infusion and withheld after Ramsey sedation scale (RSS) reached 4 to 5 in the D group. In the P group, we used a computer- 
controlled infusion to target a concentration of $0.5 \mu \mathrm{g} / \mathrm{ml}$ at the effect site, and then to increase the target concentration by $0.5 \mu \mathrm{g} / \mathrm{ml}$ every $5 \mathrm{~min}$ until satisfactory sedation was achieved. We recorded the vital signs, segmental Narcotrend EEG power spectra and arterial blood analysis of both groups before sedation (baseline), immediately after drug administration ended, 5, 10 and $15 \mathrm{~min}$. All RSS were rated by two doctors 9,10 .

Statistical analysis

All statistical analyses were carried out using the IBM commercial software package, SPSS version 19 (Armonk, NY, USA). Data were expressed as mean \pm standard deviation. For comparison within the group, parametrical data were statistically analyzed using the paired $t$-test, constituent ratio date were analyzed using paired rank-sum test. For comparison between the group, parametrical data were analyzed using two independent sample $t$-test, constituent ratio date were analyzed using two independent samples ranksum test. Narcotrend EEG power spectra at different time were compared by analysis of variance. Wilcoxon signed rank sum test and Spearman's rank correlation analysis were used to analyze the association of factors with the EEG. Differences in all analyses were considered statistically significant if a $p$ value was less than 0.05 .

\section{Results}

General data

There was no differences $(P>0.05)$ in the changes of vital signs between the two groups when satisfactory sedation was established (Table 1).

Drug administration was smooth ((RSS $4-5$ was reached) in all 47 patients. No adverse events or significant changes in the vital parameters were observed (as shown in tables 1 - 2 and figure 1). Mean values for mean arterial pressure (MAP), respiratory rate (RR), respiratory rate (RR), and $\mathrm{ETCO}_{2}$ (end-tidal $\mathrm{CO}_{2}$ ) were- $6.71 \pm 13.95 \mathrm{mmHg},-2.79 \pm 3.93,-15.43 \pm 12.84$ and $2.95 \pm 3.33$, respectively, for $\mathrm{D}$ group and $-14.36 \pm 5.80,-1.57 \pm 2.77,-12.60 \pm 14.10$ and $4.68 \pm 2.33$, respectively, for the P group. Noninvasive systolic blood pressure $\triangle \mathrm{BPs} \backslash$ and diastolic blood pressure $\triangle \mathrm{BPd} \rrbracket$, respiratory rate (RR), respiratory rate (RR), $\mathrm{ETCO}_{2}$ (end-tidal $\mathrm{CO}_{2}$ ) and $\mathrm{SpO}_{2}$ were shown in Figure 1.

Comparison of EEG between immediate after drug administration ended and the basic value.

As shown in Fig.2, after satisfactory sedation was achieved (RSS 4-5), comparing with the baseline, , the percentages of beta was decreased $\mathbb{P} \otimes 0.05 \bigotimes$ in both group $\mathrm{D}$ and $\mathrm{P}$. However, in group $\mathrm{D} \otimes$ the percentages

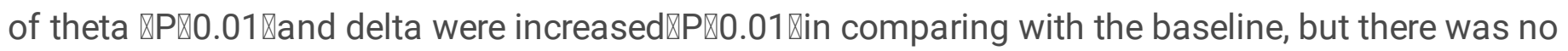
change in group P. But the percentages of alpha was increased $\mathbb{P}[0.001$ \in group P. Meanwhile, propofol sedation was characterized with alpha wave, and dexmedetomidine sedation was characterized with theta wave after satisfactory sedation was achieved (RSS 4-5). 
Comparison of EEG between after drug administration ended and waked patients up.

As shown in Fig. 3, after satisfactory sedation was achieved (RSS 4 - 5), the patients were roused, compared with the spectra after drug administration ended, the percentages of alpha power, beta power and theta power were increased and the percentages of delta power were decreased in group $D(p<$ 0.001). The percentages of alpha power were decreased, the percentages of beta power were increased in group $P(p \otimes 0.05)$. However, there was no significant difference in the segmental power spectrum of theta and delta in group $P(p>0.05)$.

Comparison of EEG at different time points in two groups at drug withdrawal $₫ 5 \min \otimes 10 \mathrm{~min} \otimes 15 \mathrm{~min}$ after drug administration ended.

To search for special neurophysiology signatures induced by dexmedetomidine and propofol, we compared the change in power percentages of alpha, beta, theta and delta at drug withhold, $5 \mathrm{~min}, 10$ min and 15 min after drug administration ended in group D and group P. As shown in Fig. 4, the percentages of alpha and theta power were decreased gradually after drug administration ended $(P<$ $0.05)$ in group $P$, while those for group $D$ were not changed $(P>0.05)$.

Relationship between electroencephalogram and the mechanism of action of dexmedetomidine and propofol.

Neurophysiology and electroencephalogram signatures of propofol- and dexmedetomidine-induced sedation are different. As shown in Fig. 4, dexmedetomidine binds the a2 adrenergic receptors to hyperpolarize locus coeruleus neurons, which decreases norepinephrine release. Propofol binds postsynaptically to GABAA receptors and GABAergic inhibitory interneurons are widely distributed throughout the cortex, thalamus, brainstem and spinal cord. Propofol induces sedation through its actions at multiple sites. The inhibitory effects of propofol disturb the highly interconnection of the thalamus and cortex and lead to the arousal of $a$ and $\beta$. By reaching the GABAergic inhibitory synapses emanating from the preoptic area onto the major arousal centers in the brainstem, propofol inhibits the excitatory arousal input from the brainstem, leading to the appearance of $\delta$ wave. By hyperpolarizing the locus ceruleus neurons, dexmedetomidine results in loss of inhibitory inputs to the pre-optic area of the hypothalamus and the pre-optic area sends GABAergic and gabenergic inhibitory projections to the major arousal centers in the midbrain and hypothalamus, leading to the appearance of $\delta$ wave. Propofol-induced $a$ and $\beta$ waves are likely resulted from decreased excitatory inputs to the cortex, but dexmedetomidine-induced $\delta$ wave is likely resulted from the disturbance in the inhibitory circuits emanating from the pre-optic area to the arousal centers. This difference in cortex and the thalamo-cortex activity suggests why patients can be aroused from dexmedetomidine-induced sedation. The wide distribution of GABAergic inhibitory pathway and the cortical activity are likely to be more profoundly inhibited by propofol compared to dexmedetomidine.

\section{Discussion}


Anesthesiologists often induce an anesthesia state best suited for the patients to have surgical or diagnostic procedure by administering selected drugs ${ }^{11,12}$. Electroencephalogram is regarded as the only brain monitor for estimating the depth and state of anesthesia ${ }^{2,13}$. Monitoring the change of EEG sectional power spectrum and the depth of anesthesia during anesthesia is important for managing anesthesia.

A growing number of evidence has proven that anesthetics with different molecular targets in the central nervous system produce diverse electroencephalograms ${ }^{11}$. Therefore, we aimed to identify the characterizations of unprocessed electroencephalograms under the sedation of propofol or dexmedetomidine, the most widely used intravenous anesthetics.

We have found the following. 1) Alpha and delta oscillations were the markers of propofol-induced sedation. Dexmedetomidine-induced sedation was characterized with delta oscillations. Although alpha oscillation could also be collected during dexmetomidine-induced sedation, compared with the coherent and continuous alpha oscillation associated with propofol, the alpha oscillations associated with dexmedetomidine were brief, episodic and susceptible. 2) When patients gradually regained consciousness by verbal stimulation, $\delta$ oscillation dissipated in the D group. Simultaneously, there were increasing numbers of $\alpha, \beta$ and $\theta$ oscillation. But the number of $\alpha$ and $\beta$ oscillations in the $P$ group was increased until $15 \mathrm{~min}$ after the discontinuation of infusion. 3) By identifying the special signatures of propofol- and dexmedetomidine-induced sedation in Narcotrend, Narcotrend could assist anesthesiologists to accurately analyze and track anesthetic effects on the brain.

Propofol, the most widely used $y$-aminobutyric acid receptor-specific agonist, is used as an induction agent and to maintain sedation and general anesthesia by its actions at multiple sites in neural circuits including the cortex, thalamus, brainstem and spinal cord. In our study, we observed that $a$ and $\delta$ oscillations were the main characterization of propofol-induced sedation. This result is in accordance with a previous study ${ }^{14}$. Delta oscillation is closely associated with the brainstem and regular and coherent delta oscillations are responses to brainstem stimulation ${ }^{15,16}$. After infusion administration, propofol rapidly reaches GABAergic inhibitory synapses on the major arousal centers in the brainstem, preventing the excitatory arousal signal coming from the brainstem, and impeding excitatory input from the brainstem to the thalamus and the cortex. On the other hand, the clinical characteristics accompanied by the appearance of delta oscillation coincides with effects of anesthetic on the brainstem, such as loss of responsiveness, loss of oculocephalic reflex, apnea and atonia ${ }^{17,18}$. We also found that there were highly coherent alpha oscillations across the front of the head during propofol-induced sedation. Alpha oscillation is closely related to the functions of the thalamic reticular nucleus that is crucial for sleep regulation ${ }^{19}$. Through enhancing the GABAergic inhibition at the thalamic reticulate nucleus, propofol enhances inhibition input from the thalamus to the cortex. Ultimately, propofol leads to hyperpolarization of the cortical pyramidal neurons ${ }^{20,21}$. Thus, it is reasonable to speculate that the coherent alpha prevented the normal communication between the thalamus and the cortex. 
The EEG characterization of dexmedetomidine-induced sedation is different from that of propofolinduced sedation. As a pre-synaptic a2 adrenergic receptor agonist, dexmedetomidine induces its sedative and anesthetic effects by hyperpolarizing the locus coeruleus neurons, leading to loss of inhibitory inputs to the pro-optic area of the hypothalamus and finally the GABAergic inhibitory projections sending from the pro-optic area of the hypothalamus to the major arousal centers in the midbrain, pons and hypothalamus, resulting in sedation. Our results showed that delta oscillation was the EEG marker during dexmedetomidine-induced sedation. When its infusion was discontinued, patients were easily aroused by verbal or tackled stimulation and the delta oscillation was dissipated and gradually replaced by $a, \beta$ and $\theta$ oscillations. The delta oscillation was similar to non-rapid eye movement (NREM) sleep stage or slow-wave sleep. The main reason may be that the molecular targets of dexmedetomidine involve the generation of NREM sleep. Compared with propofol, patients with dexmedetomidine infusion were easier to recover from sedation by verbal or tackled stimulation.

Although delta oscillation could be found in both propofol- and dexmedetomidine-induced sedations, the delta and alpha oscillations in propofol-induced sedation were highly coherent and continuous but the delta oscillations caused by dexmedetomidine were brief, episodic and susceptible. This phenomenon may explain why sedation with propofol produces a deeper state of sedation from which patients cannot be aroused easily. Furthermore, the different sedative states between propofol and dexmedetomidine may be due to their different molecular targets. Propofol exerts its effects by binding post-synaptically to $\mathrm{GABA}_{\mathrm{A}}$ receptors and GABAergic inhibitory receptors are widely distributed throughout the cortex, thalamus, brainstem and spinal cord. Dexmedetomidine indirectly sends GABAergic inhibitory signals to the major arousal centers in the midbrain, pons and hypothalamus. Oluwaseun Akeju et al. have shown that during dexmedetomidine-induced unconsciousness, cortico-cortical functional connectivity remains intact but thalamo-cortical functional connectivity is disrupted ${ }^{22}$. Hence, the brief, episodic and susceptible delta oscillation may reflect a limited and lower level of disturbances in neuronal activity under dexemedetomidine sedation compared to propofol sedation. In our study, $15 \mathrm{~min}$ after the drug infusion was discontinued, the alpha and delta oscillations in the propofol group gradually disappeared. However, there were no changes in the electroencephalogram in the dexmedetomidine group. The phenomenon may also suggest that propofol-induced sedations are likely to have more profound inhibitory effects compared to dexmedetomidine-induced sedation. The result is also a reminder to anesthetists that anoxia and apnea may still occur due to the inhibitory effect even 15 min after propofol has been discontinued.

Our results are consistent with that of Oluwaseun Akeju et al ${ }^{23}$ who used whole brain EEG. Whole brain EEG is widely used in scientific research. However, because of its complex operations, a large amount of information and complex analysis, its use in clinical settings is limited. The Narcotrend used in our study is the most important tool for evaluating the depth of anesthesia, for which the most common method is EEG index, which is simple and easy to use. We found that the EEG waveforms induced by dexamethasone and propofol were significantly different on the Narcotrend at the time of sedation and awakening. We also found that Narcotrend had a better correlation with the depth of sedation caused by 
propofol than the traditional sedation score. At the same depth of RSS sedation caused by propofol, dexmedetomidine-induced Narcotrend indices were lower, and Narcotrend indice were not stable with the change in arousal. It is not suitable for monitoring dexmedetomidine-induced sedation in patients. Our study provides important clinical data for the correct use of Narcotrend in clinical settings.

\section{Abbreviations}




\begin{tabular}{|c|c|}
\hline Abbreviations & Full name \\
\hline ASA & American Society of Anesthesia \\
\hline EEG & Electroencephalogram \\
\hline CSEA & Combined spinal-epidural anesthesia, \\
\hline DEX & Dexmedetomidine \\
\hline PRO & Propofol \\
\hline NT & Narcotrend \\
\hline NTI & Narcotrend Index \\
\hline NTS & Narcotrend Stage \\
\hline BIS & BISpectral index \\
\hline RSS & Ramsay sedation scale \\
\hline $\mathrm{TCl}$ & Target-controlled infusion \\
\hline FDA & Food and Drug Administration \\
\hline BMI & Body Mass Index \\
\hline $\mathrm{HR}$ & Heart Rate \\
\hline RR & Respiration Rate \\
\hline $\mathrm{PCO}_{2}$ & Partial pressure of Carbon Dioxide \\
\hline GABA & Gamma-aminobutyric acid \\
\hline a2AR & A 2 adrenergic receptors \\
\hline REM & Rapid Eye Movement Sleep \\
\hline NREM & Non-rapid eye movement sleep \\
\hline a & Alpha \\
\hline$\beta$ & Beta \\
\hline$\theta$ & Theta \\
\hline$\delta$ & Delta \\
\hline
\end{tabular}

\section{Declarations}

Acknowledgements

Ethics declarations 
Ethics approval and consent to participate

The experimental protocol for patient samples studies was reviewed and approved by the Ethics Review Committee at Sun Yat-Sen University.

The study was conducted under good clinical practice guidelines and was registered in Chinese Clinical Trial Registry (ChiCTR) (www.chictr.org.cn, registration number: ChiCTR1900021562)

Availability of data and materials

All data generated or analyzed during this study are included within the article.

Conflict of interest

There are no potential conflicts of interest to be disclosed.

Funding

Not applicable

Authors' information

Mao Zhou (MMed) Department of Anesthesiology, Sun Yat-Sen Memorial Hospital, Sun Yat-Sen University, Guangzhou, 510289, Guangdong, China.

shanhaidan@163.com

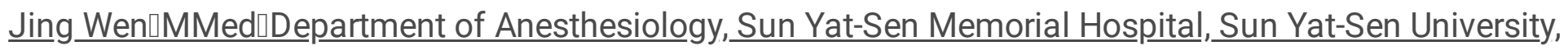
Guangzhou, $\underline{510289}$, Guangdong, China.

wenj8@@mail2.sysu.edu.cn

Jun Peng $₫$ M.D.『Department of Anesthesiology, Sun Yat-Sen Memorial Hospital, Sun Yat-Sen University, Guangzhou, 510289, Guangdong, China.

pengjun2@mail.sysu.edu.cn

Corresponding authors

Zhiyi Zuo \M.D.『Department of Anesthesiology, University of Virginia, Charlottesville, Virginia USA zz3c@virginia.edu.

Shuling Peng $₫$ M.D. $\varangle$ Department of Anesthesiology, Sun Yat-Sen Memorial Hospital, Sun Yat-Sen University, Guangzhou, 510289, Guangdong, China.

pslmzk@aliyun.com 
Author contribution:

Author name: Mao Zhou, this author performed research and collected the data.

Author name: Jing Wen, this author wrote the manuscript and collected the data.

Author name: Jun Peng, this author assisted in revising the manuscript.

Author name: Zhiyi Zuo, this author designed the research and revised the manuscript.

Author name: Shuling Peng, this author designed the research and revised the manuscript.

Mao Zhou and Jing Wen contributed equally to this paper.

\section{References}

1. Britton, J.W, Frey LC, Hopp JL, Korb P. Electroencephalography (EEG): An Introductory Text and Atlas of Normal and Abnormal Findings in Adults, Children, and Infants. 2016, Chicago: American Epilepsy Society.

2. 2. Escallier, K.E. Monitoring the brain: processed electroencephalogram and peri-operative outcomes. Anaesthesia, 2014. 69(8): p. 899-910.

3. Cascella, M. Mechanisms underlying brain monitoring during anesthesia: limitations, possible improvements, and perspectives. Korean J Anesthesiol, 2016. 69(2): p. 113-20.

4. 4. Bydder, M, Zaaraoui W, Ridley B, Soubrier M. Dynamic (23)Na MRI - A non-invasive window on neuroglial-vascular mechanisms underlying brain function. Neuroimage, 2019. 184: p. 771-780.

5. Ching, S, E.N. Brown, Modeling the dynamical effects of anesthesia on brain circuits. Curr Opin Neurobiol, 2014. 25: p. 116-22.

6. 6. Wagshul, M.E, Lucas M, Ye K. Multi-modal neuroimaging of dual-task walking: Structural MRI and fNIRS analysis reveals prefrontal grey matter volume moderation of brain activation in older adults. Neuroimage, 2019. 189: p. 745-754.

7. Kasuya, Y, Govinda R, Rauch S, Mascha EJ. The correlation between bispectral index and observational sedation scale in volunteers sedated with dexmedetomidine and propofol. Anesth Analg, 2009. 109(6): p. 1811-5.

8. 8. Garrity, A.G, Botta S, Lazar SB, Swor E. Dexmedetomidine-induced sedation does not mimic the neurobehavioral phenotypes of sleep in Sprague Dawley rat. Sleep, 2015. 38(1): p. 73-84.

9. Otto, K.A, Cebotari S, Höffler HK. Electroencephalographic Narcotrend index, spectral edge frequency and median power frequency as guide to anaesthetic depth for cardiac surgery in laboratory sheep. Vet J, 2012. 191(3): p. 354-9.

10. Weber, F., H. Hollnberger, J. Weber, Electroencephalographic Narcotrend Index monitoring during procedural sedation and analgesia in children. Paediatr Anaesth, 2008. 18(9): p. 823-30. 
11. Kaskinoro, K, Maksimow A, Georgiadis S, Långsjö J. Electroencephalogram reactivity to verbal command after dexmedetomidine, propofol and sevoflurane-induced unresponsiveness. Anaesthesia, 2015. 70(2): p. 190-204.

12. 12. Purdon, P.L, Sampson A, Pavone KJ. Clinical Electroencephalography for Anesthesiologists: Part I: Background and Basic Signatures. Anesthesiology, 2015. 123(4): p. 937-60.

13. 13. Fahy, B.G, D.F. Chau. The Technology of Processed Electroencephalogram Monitoring Devices for Assessment of Depth of Anesthesia. Anesth Analg, 2018. 126(1): p. 111-117.

14. 14. Kaskinoro, $K$, Silfverhuth $M$, Långsjö J, Kaskinoro K. Directional connectivity between frontal and posterior brain regions is altered with increasing concentrations of propofol. PLoS One 2014 Nov 24;9(11):e113616.

15. Kiss, T, Feng J, Hoffmann WE, Shaffer CL. Rhythmic theta and delta activity of cortical and hippocampal neuronal networks in genetically or pharmacologically induced N-methyl-D-aspartate receptor hypofunction under urethane anesthesia. Neuroscience, 2013. 237: p. 255-67.

16. 16. Akeju, O, Pavone KJ, Westover MB, Vazquez Ret. A comparison of propofol- and dexmedetomidine-induced electroencephalogram dynamics using spectral and coherence analysis. Anesthesiology, 2014. 121(5): p. 978-89.

17. Brown, E.N, R. Lydic, N.D. Schiff, General anesthesia, sleep, and coma. N Engl J Med, 2010. 363(27): p. 2638-50.

18. 18. Babadi, B. A review of multitaper spectral analysis. IEEE Trans Biomed Eng, 2014. 61(5): p. 155564.

19. Bagshaw, A.P, Hale JR, Campos BM, Rollings DT, Sleep onset uncovers thalamic abnormalities in patients with idiopathic generalised epilepsy. Neuroimage Clin, 2017. 16: p. 52-57.

20. 20. Purdon, P.L, Pierce ET, Mukamel EA, Prerau MJ. Electroencephalogram signatures of loss and recovery of consciousness from propofol. Proc Natl Acad Sci U S A, 2013. 110(12): p. E1142-51.

21. Drummond, J.C, Brann CA, Perkins DE. A comparison of median frequency, spectral edge frequency, a frequency band power ratio, total power, and dominance shift in the determination of depth of anesthesia. Acta Anaesthesiol Scand, 1991. 35(8): p. 693-9.

22. 22. Ching, S, E.N. Brown. Modeling the dynamical effects of anesthesia on brain circuits. Curr Opin Neurobiol, 2014. 25: p. 116-22.

23. Akeju, O, Westover MB, Pavone KJ, Sampson AL. Effects of sevoflurane and propofol on frontal electroencephalogram power and coherence. Anesthesiology. 2014 Nov;121(5):990-8.

\section{Tables}

Table 1. Comparison of gender, age and BMI between two groups. 


\begin{tabular}{lccccc}
\hline & Variate & D group & P group & Statistic & $P$ \\
\hline Gender & female & $17 \llbracket 47.2 \square$ & $19 \llbracket 52.8 \square$ & $X^{2}=0.908$ & 0.341 \\
& male & $7 \llbracket 63.6 \square$ & $4 \llbracket 36.4 \square$ & & \\
Age & $` \mathrm{x} \pm \mathrm{S}$ & $41.04 \pm 10.123$ & $41.43 \pm 12.460$ & $t=0.119$ & 0.906 \\
BIM & $` \mathrm{x} \pm \mathrm{S}$ & $22.42 \pm 1.779$ & $21.77 \pm 1.823$ & $t=1.173$ & 0.248 \\
\hline
\end{tabular}

Date are represented as mean $\pm \mathrm{SD}$ of at least three independent experiments.

Table2 Comparison of vital signs between two groups when sedation satisfaction was achieved

\begin{tabular}{lccc}
\hline Variables & Group D & Group P & $P$ \\
\hline$\triangle \mathrm{MAP}$ & $-6.71 \pm 13.95$ & $-14.36 \pm 5.80$ & 0.062 \\
$\triangle \mathrm{RR}$ & $-2.79 \pm 3.93$ & $-1.57 \pm 2.77$ & 0.353 \\
$\triangle \mathrm{HR}$ & $-15.43 \pm 12.84$ & $-12.60 \pm 14.10$ & 0.578 \\
$\triangle \mathrm{PaCO}_{2}$ & $2.95 \pm 3.33$ & $4.68 \pm 2.33$ & 0.116 \\
\hline
\end{tabular}

Vital signs were recorded at least three independent experiments. Date are represented as mean $\pm \mathrm{SD}$ of. ${ }^{*} p<0.05, * * p<0.01$

\section{Figures}



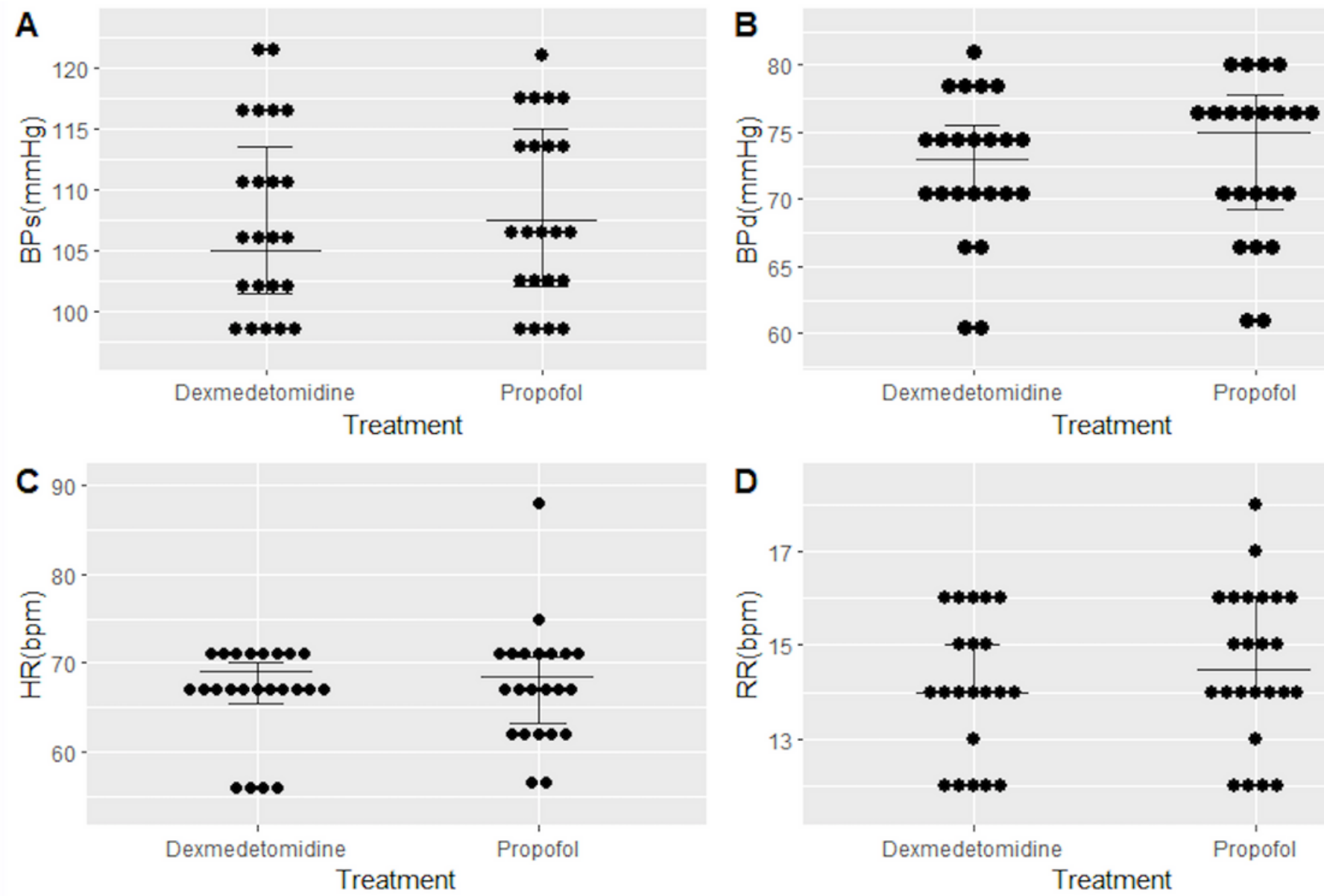

Dexmedetomidine

Treatment
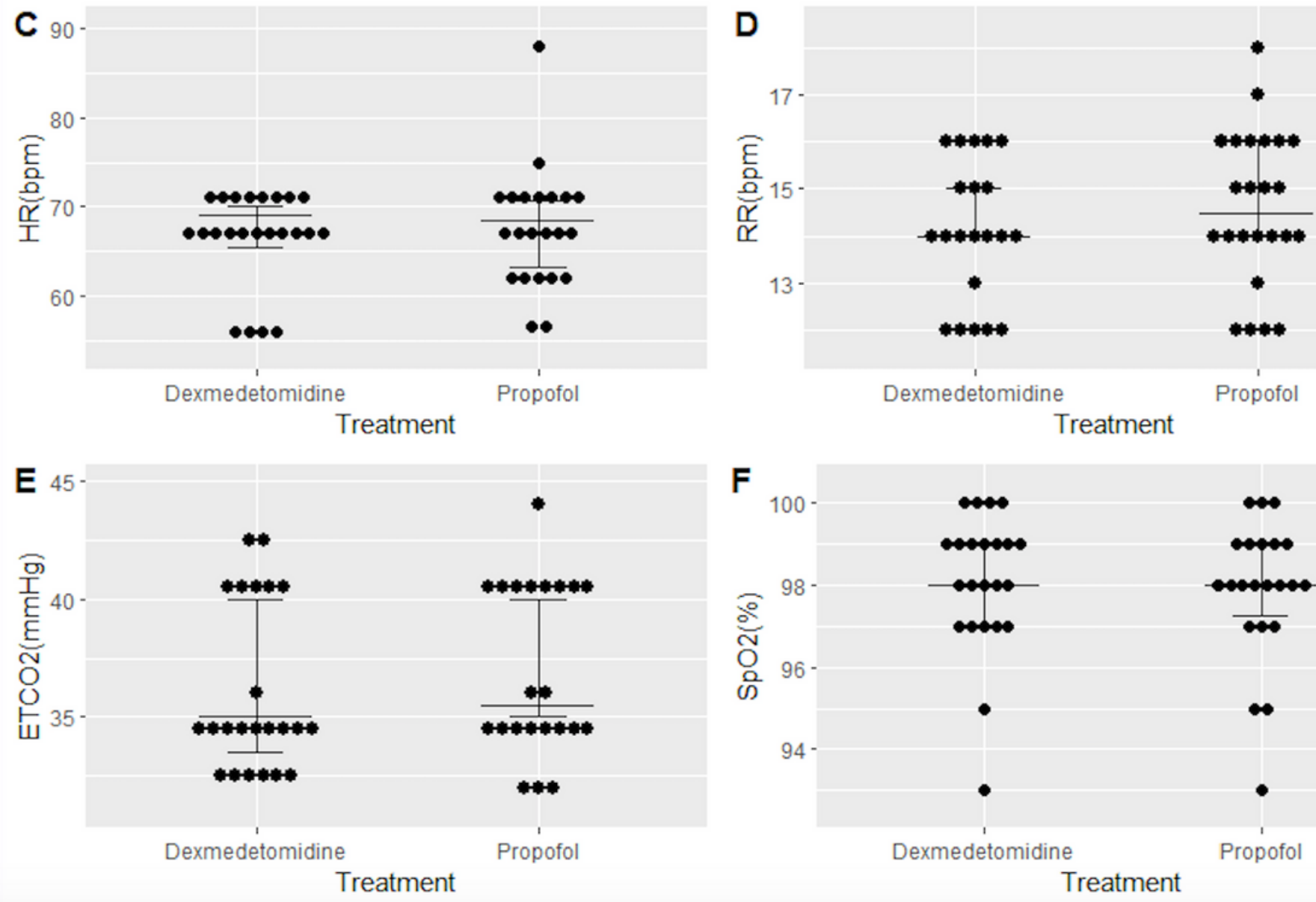

\section{Figure 1}

Noninvasive systolic blood pressure $₫ \mathrm{BP} \triangle \mathrm{s}$ and diastolic blood pressure $₫ \mathrm{BP} \mathrm{d} \rrbracket$, respiratory rate (RR), heart rateХHR囚, ETCO2 (end-tidal co2) and Spo2. Spo2 and ETco2 were obtained when $1.5 \mathrm{~L} / \mathrm{min}$ oxygen via nasal cannula was given. Data are shown with mean, $95 \%$ confidence interval and individual plots. Vital signs were recorded at least three independent experiments. 


\section{Status $\quad$ Normal $\square$ discontinue}

A

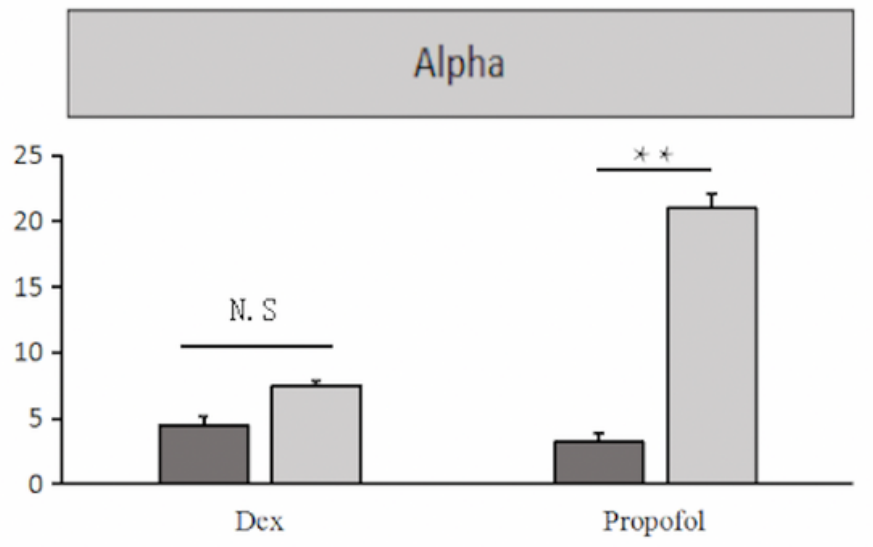

C

\section{Delta}

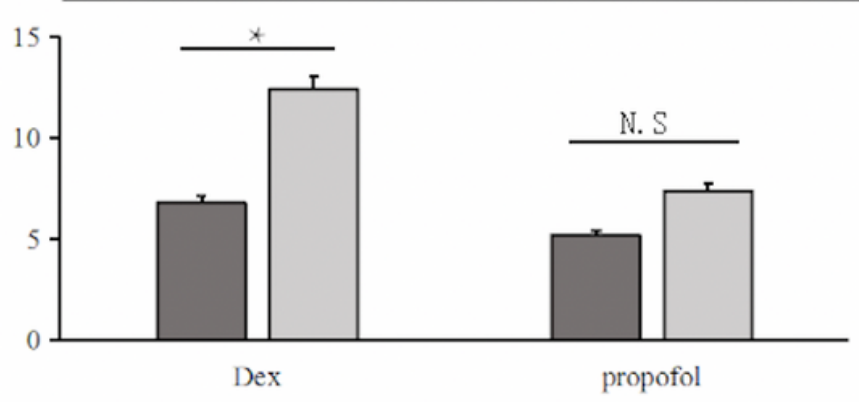

B

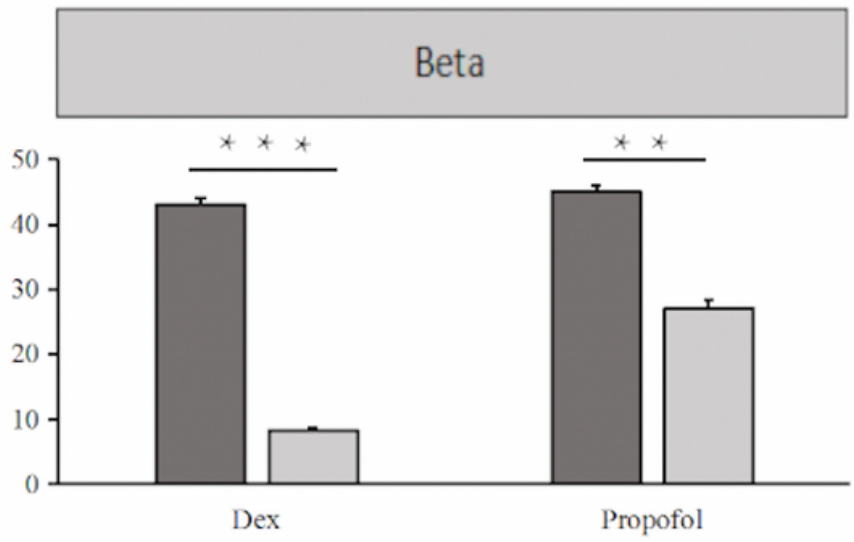

D
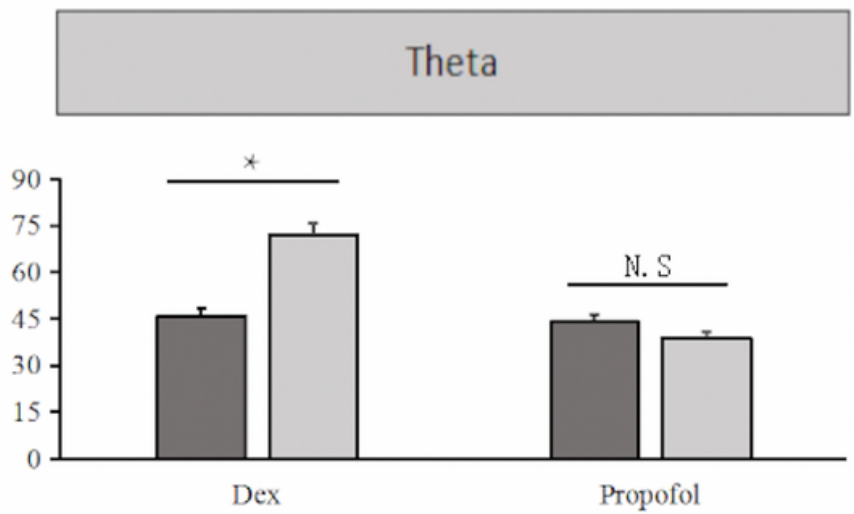

\section{Figure 2}

The percentages of alpha power, beta power, theta power and delta power at satisfactory sedation and immediately after drug administration ended in two groups. Compared with the baseline, the percentages of alpha was increased, the percentages of beta was decreased in both groups. In group DQthe percentages of theta and delta were increased in comparing with the baseline, but there was no change in

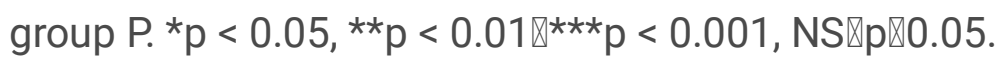


A

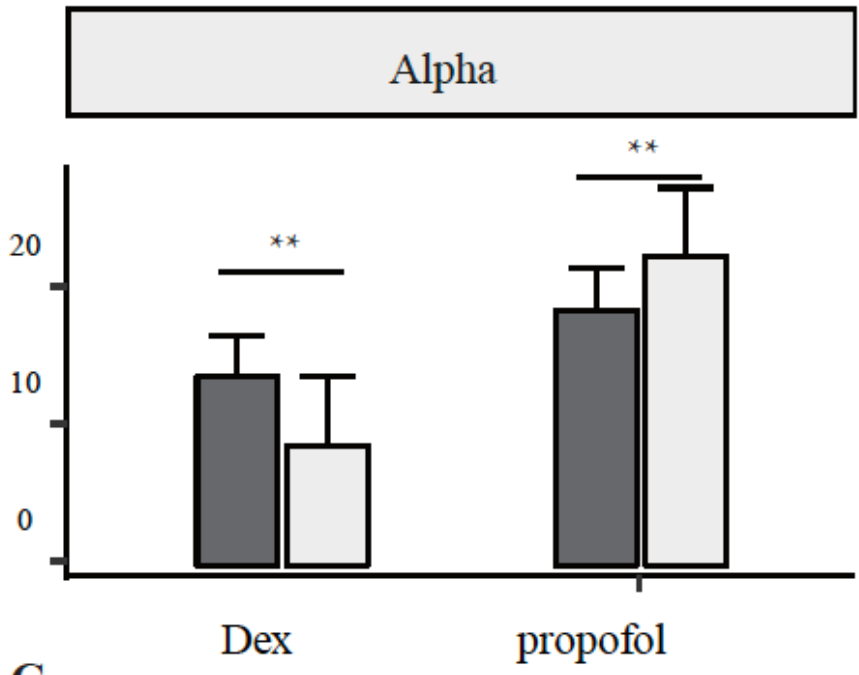

C
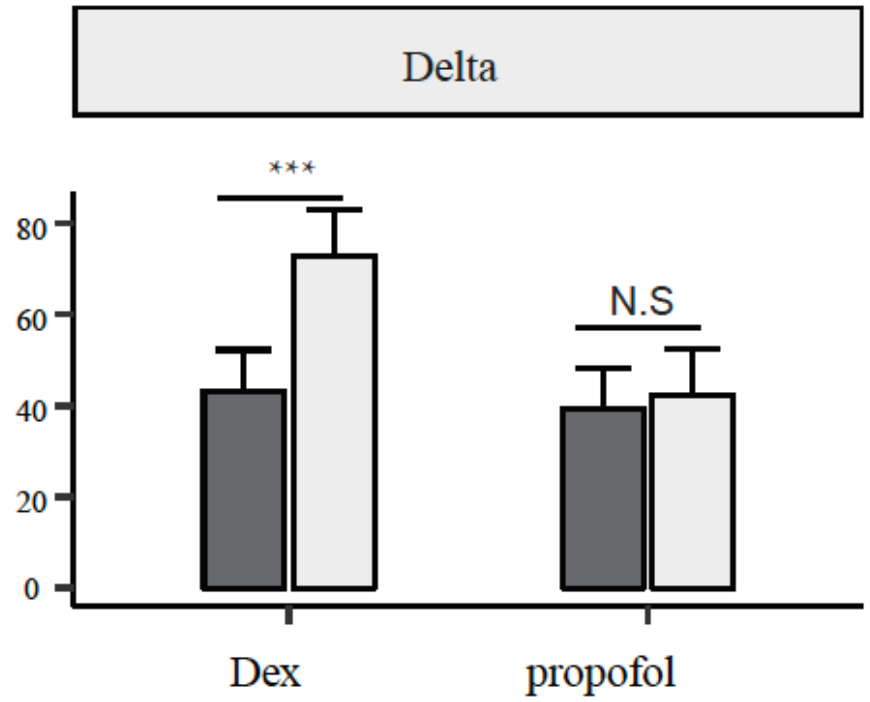

B
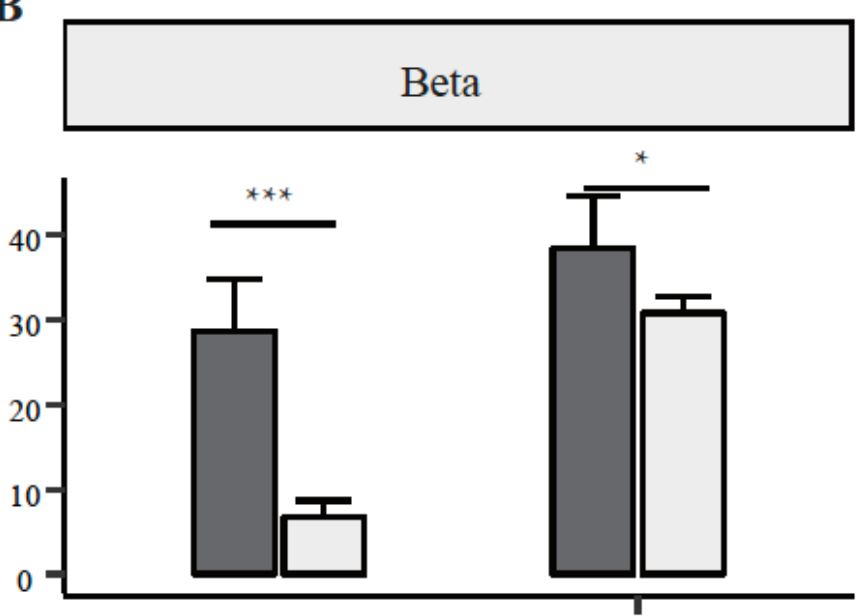

Dex
D

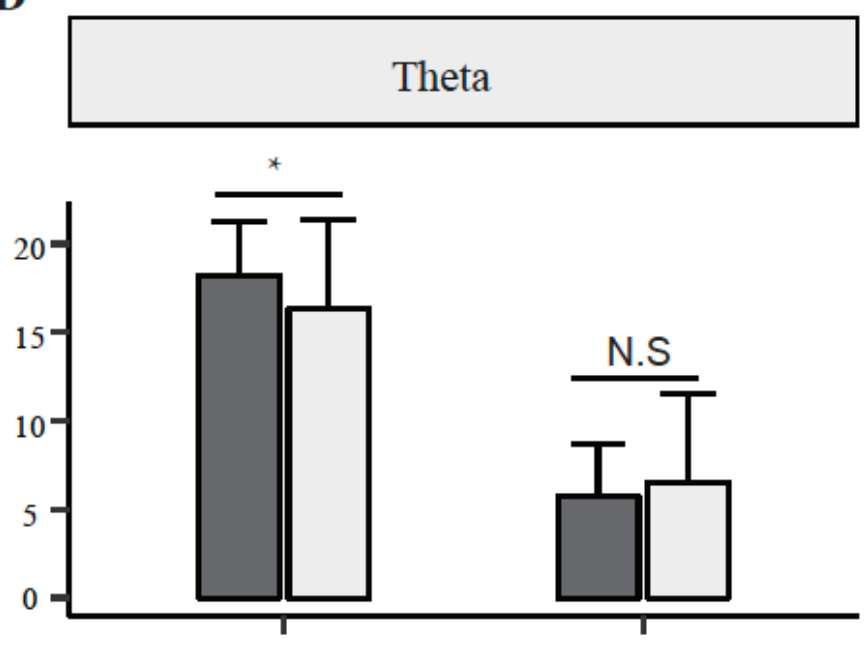

Dex

propofol

\section{Figure 3}

The percentages of alpha power, beta power, theta power and delta power at after drug administration ended and waked patients up. Compared with the spectra after drug administration ended when patients were roused, the percentages of alpha power, beta power and theta power were increased and the percentages of delta power were decreased in group $D$. There was no significant difference in the segmental power spectrum of group P. ${ }^{\star} p<0.05$, ${ }^{\star \star} p<0.01 \rrbracket \star \star \star p<0.001$ 

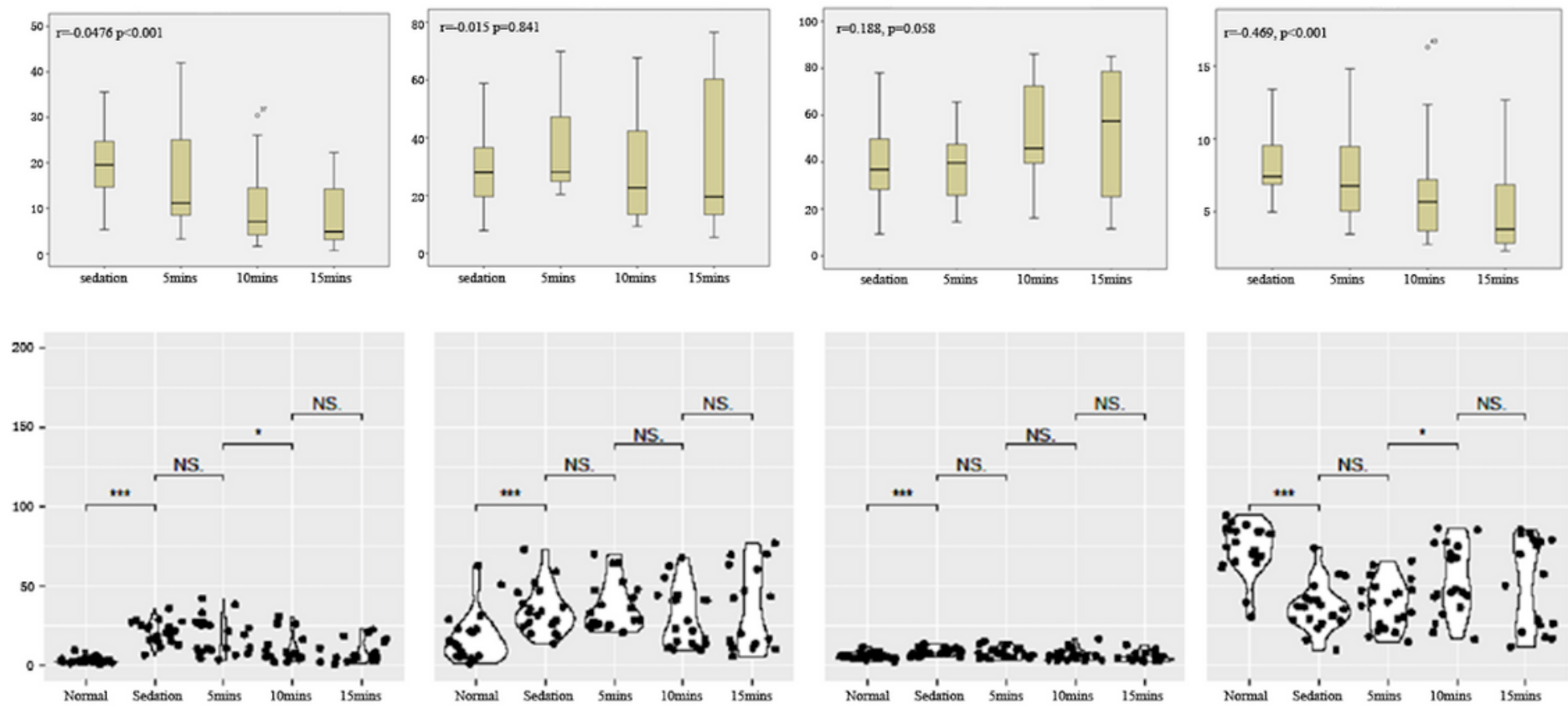

Propofol
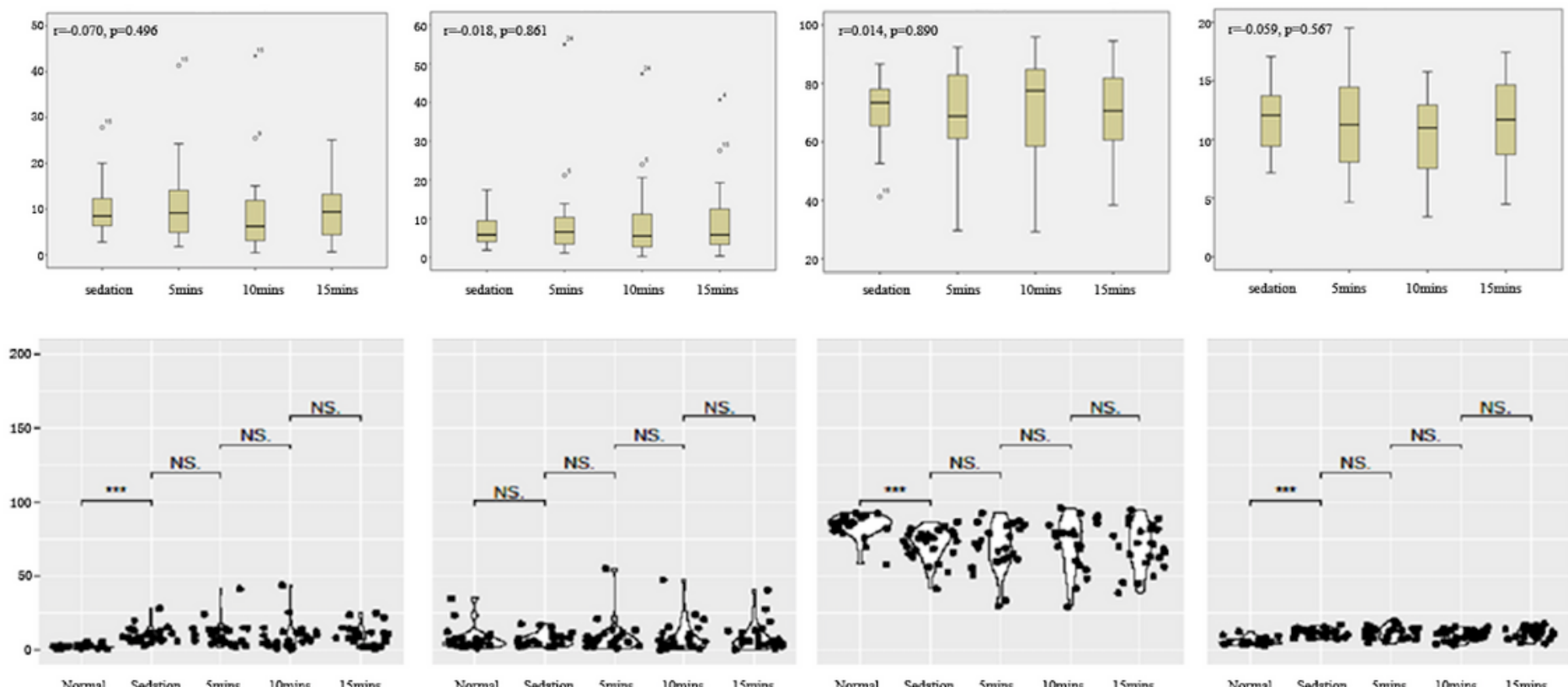

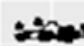

Normal Sedarion 5mins 10 mins 15 mins

Normal Sedation 5 mins 10 mins $15 \operatorname{mins}$

\section{Figure 4}

The power percentages of alpha, beta, theta, and delta at the four time points: at drug withhold, 5 min, 10 min, and 15 min after drug withhold, compared with the segmented EEG power spectrum at baseline. Immediately after drug withhold, the percentage of alpha power was increased and that of beta power was decreased in both groups $(P<0.05)$; the percentage of theta and delta power was increased in the $D$ group while those for the $P$ group were not changed $(P>0.05)$. In the $P$ group, the percentages of alpha 
and theta power were decreased gradually after drug withhold $(P<0.05)$. Immediately after drug withhold, the percentages of alpha and beta power in the $D$ group were lower than those in the $P$ group and theta and delta power percentages in the $\mathrm{D}$ group were all higher than those in $\mathrm{P}$ group. Vital signs were recorded at least three independent experiments. ${ }^{*} p<0.05$, ${ }^{* *} p<0.01 \rrbracket * \star * p<0.001 \rrbracket N S \rrbracket p \rrbracket 0.05$.

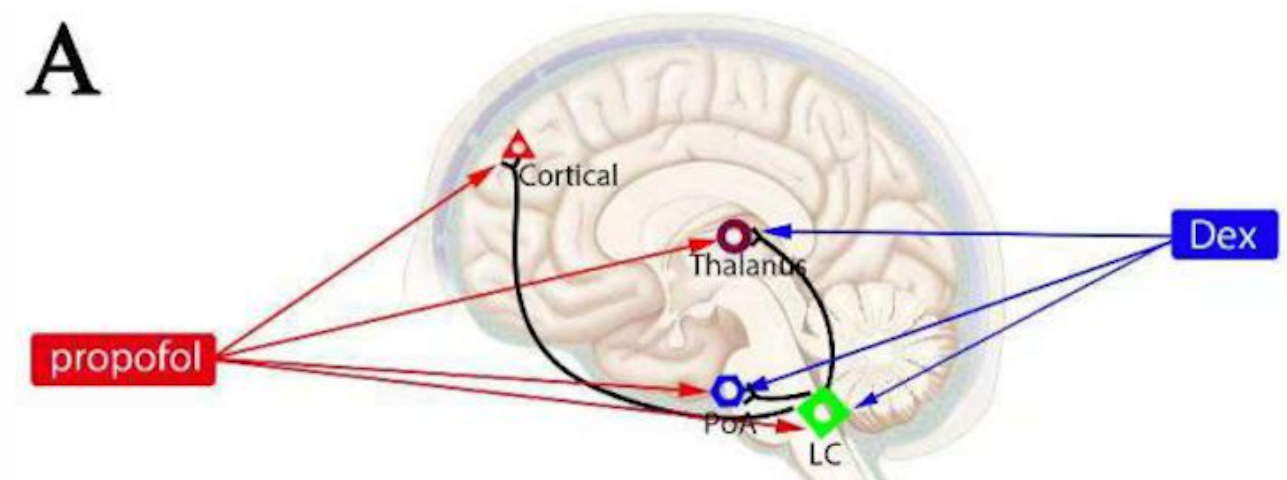

B

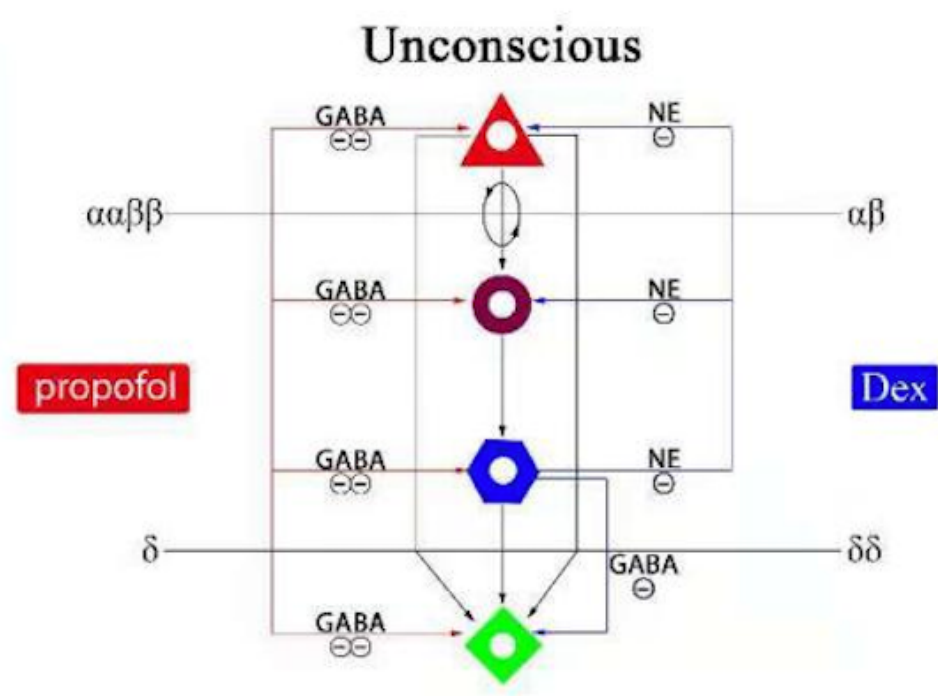

C

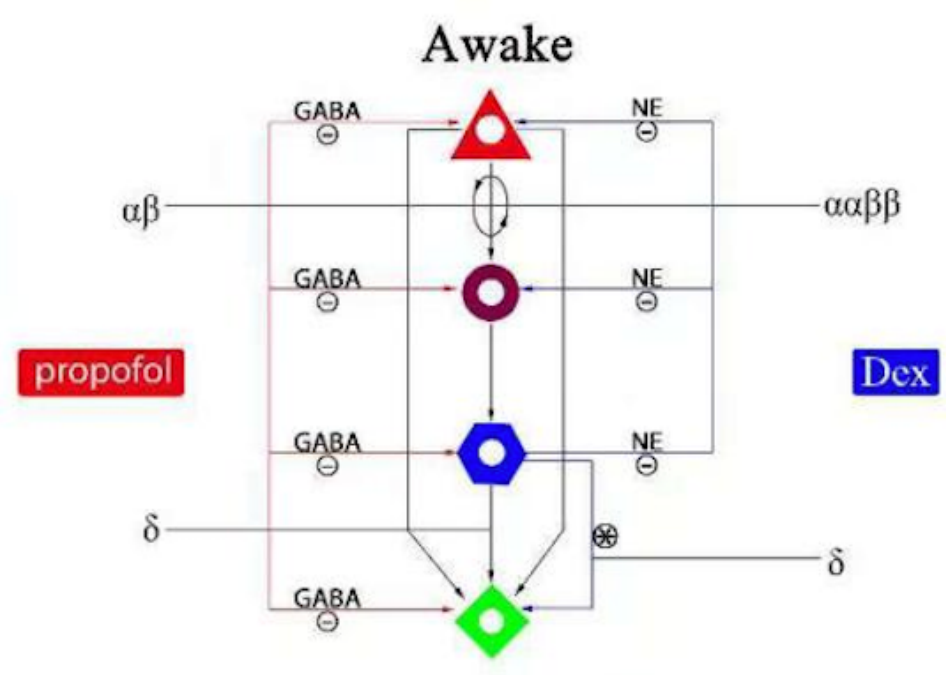

Figure 5 
Neurophysiology and electroencephalogram signatures of propofol- and dexmedetomidine-induced sedation. A. Propofol binds post-synaptically to GABAA receptors and GABAergic inhibitory interneurons are widely distributed throughout the cortex, thalamus, brainstem and spinal cord. Propofol induces sedation through its actions at multiple sites. Dexmedetomidine binds the a2 adrenergic receptors to hyperpolarize locus coeruleus neurons, which decreases norepinephrine release. B. The inhibitory effects of propofol disturb the highly interconnection of the thalamus and cortex and lead to the arousal of $\mathrm{a}$ and $\beta$. By reaching the GABAergic inhibitory synapses emanating from the pre-optic area onto the major arousal centers in the brainstem, propofol inhibits the excitatory arousal input from the brainstem, leading to the appearance of $\delta$ wave. By hyperpolarizing the locus ceruleus neurons, dexmedetomidine results in loss of inhibitory inputs to the pre-optic area of the hypothalamus and the pre-optic area sends GABAergic and gabenergic inhibitory projections to the major arousal centers in the midbrain and hypothalamus, leading to the appearance of $\delta$ wave. C. Propofol-induced $a$ and $\beta$ waves are likely resulted from decreased excitatory inputs to the cortex, but dexmedetomidine-induced $\delta$ wave is likely resulted from the disturbance in the inhibitory circuits emanating from the pre-optic area to the arousal centers. This difference in cortex and the thalamo-cortex activity suggests why patients can be aroused from dexmedetomidine-induced sedation. The wide distribution of GABAergic inhibitory pathway and the cortical activity are likely to be more profoundly inhibited by propofol compared to dexmedetomidine. 